\title{
Modeling and Visualization of Knitwear
}

\author{
Eduard Gröller, Member, IEEE Computer Society, René T. Rau and Wolfgang Straßer
}

\begin{abstract}
Visualization and modeling of textile materials has already been investigated in detail in the computer graphics literature. Most of the work, however, concentrates on woven fabrics. In this paper we present a novel approach to the modeling and rendering of knitwear. After the topological specification of a knitting pattern a subdivision into basic elements is done. The yarn microstructure within basic elements is approximated by volume data sets. The repetitive structure of knitted fabrics allows an efficient rendering technique. Resulting images are given that demonstrate the feasibility of our approach.
\end{abstract}

Index Terms - Knitwear, volume visualization, textile modeling.

\section{INTRODUCTION}

$\mathrm{R}$ EALISTIC representation and rendering of textiles is important for various fields of industry and research, e.g., fashion design, interior design and human animation. Textiles are made up of yarns which in turn consist of elementary fibers. There is a wide variety of possibilities to combine yarns, each defining a different type of textile structure. Some typical textile structures are woven materials, knitwear and lace fabrics. Despite these various textile types most research literature in computer graphics deals only with woven materials. Some work has been done on modeling and rendering the macrostructure of woven materials, e.g., [4], [12]. In [4] a physically-based technique for predicting the drape of woven fabrics is investigated. Interacting particles define small-scale interactions. The correct macroscopic behavior is produced by computationally aggregating these interactions. Other work deals with the interaction of light with textile surfaces. A lighting model for rendering the peculiar gloss of cloth objects is presented in [14]. Considering microscopic fabric features such as fiber cross-section or weave (warp, weft) the authors come up with an anisotropic lighting model consisting of specular reflection, internal reflection, and diffuse reflection. It is, however, assumed that cloth is viewed from a distance, so that single yarns are not distinguishable.

For the virtual design there is a need in textile industry to visualize not only woven materials but knitwear as well. We realized this in collaboration with an industrial partner. This paper presents an approach for realistic representation of knitted fabrics and thread structure. Our technique models the yarn microstructure as 3D data to allow a close-up inspection of the model. Previous methods for textile design are based on 2D

Eduard Gröller is with Institut für Computergraphik, Technische Universität Wien, Karlsplatz 13/186/2, A-1040 Wien, Austria; e-mail: groeller@cg.tuwien.ac.at.

René Rau and Wolfgang Straßer are with WSI/GRIS, Universität Tübingen, Auf der Morgenstelle 10, D-72076 Tübingen, Germany; e-mail: rrau or strasser@gris.informatik.uni-tuebingen.de. texture mapping to express cloth texture. Typically the texture is gained by scanning real textile materials which is then mapped onto surfaces. Close-up inspection is limited by texture resolution and no insight into the real 3D microstructure of the material can be achieved. This is especially disadvantageous when considering knitted fabrics which often are characterized by rather pronounced 3D microshapes. Visualizing the 3D structure of knitwear explicitly allows a fast virtual design of knitted fabrics with the possibility to easily change various parameters, e.g. loop width, color, yarn type, yarn thickness, and arrangement of knitted stitch types. Rendering the 3D structure of knitwear enables low-cost rapid prototyping without the need to produce real samples of novel knitted fabrics.

Rendering of human hair ([3], [5],[13]) is somewhat related to our task in that it also involves the modeling of fibrelike objects. Most approaches for hair modeling represent the geometry of single hairs or strands of hairs explicitely. Our method, on the other hand, is more in the spirit of the method described in [7]. There a technique for the rendering of furry surfaces is introduced. Rendering primitives are volume densities with anisotropic lighting behavior. Instead of an explicit representation of individual hairs the authors use so called texels which are threedimensional arrays of parameters approximating visual properties of collections of microsurfaces. The parameters stored at each location of a texel are: density, representative orientation of microsurfaces, and reflectance function. Rendering furry objects includes creation of texels, mapping of texels into world space, the intersection of rays with texels, and the computation of the lighting model. Knitting yarn has a fine microstructure where single fibers are not perceivable by themselves but provide an important contribution to the overall visual impression of the yarn structure. Similar to the idea of introducing texels [7], we do not model the geometry of the yarn microstructure explicitely but use volume data sets to represent collections of fibers simultaneously. Therefore rendering time is independent from the geometric complexity of the yarn structure. Modeling of phenomena intermediate between shape and texture has also been done in [9].

Section 2 describes the topological construction of knitted fabrics. In Section 3 modeling of the yarn microstructure as volume data is explained. Section 4 investigates efficient rendering approaches of knitted fabrics by exploiting various periodicity properties of knitwear. Section 5 discusses resulting images and Section 6 outlines future work.

\section{A Simple Knitwear Model}

Simple knitwear patterns are made up of only two types of loops (R-loop or plain stitch and L-loop or reverse stitch) and therefore have a highly repetitive structure. A knitwear pattern is constructed row by row where each row consists of a series of consecutive loops. For each loop of the previous row a new loop on the current row is built by pulling the knitting yarn through 
the loop of the previous row either from front-to-back (L-loop for left loop) or back-to-front (R-loop for right loop). R-loops and L-loops are shown in Fig. 1. Notice the view-dependent definition of the type of a loop, i.e., the front view of an R-loop is the back view of an L-loop and viceversa.
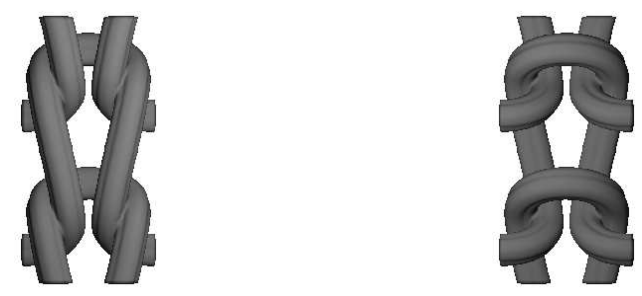

Fig. 1. R-loop (plain stitch) and L-loop (reverse stitch).

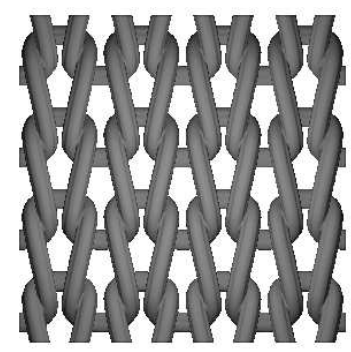

\begin{tabular}{|c|c|c|c|}
\hline $\mathbf{R}$ & $\mathbf{R}$ & $\mathbf{R}$ & $\mathbf{R}$ \\
\hline $\mathbf{R}$ & $\mathbf{R}$ & $\mathbf{R}$ & $\mathbf{R}$ \\
\hline $\mathbf{R}$ & $\mathbf{R}$ & $\mathbf{R}$ & $\mathbf{R}$ \\
\hline $\mathbf{R}$ & $\mathbf{R}$ & $\mathbf{R}$ & $\mathbf{R}$ \\
\hline
\end{tabular}
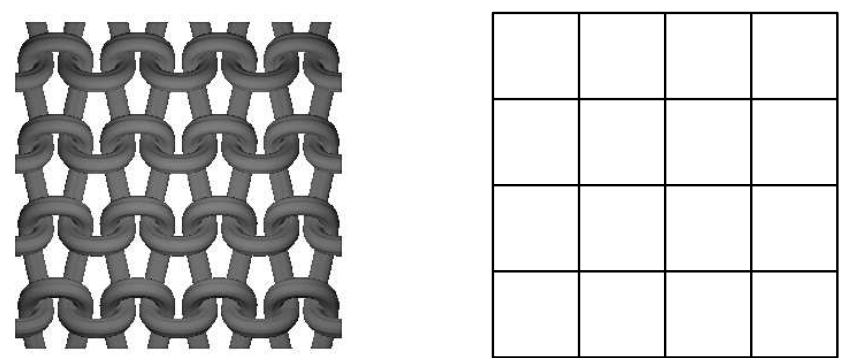

Fig. 2. RL-knitted fabric, front view and back view.

Fig. 2 and 3 illustrate simple knitted fabrics by giving the topology of the thread structure and an abstract representation. The abstract representation specifies the types of loops the knitwear pattern is made of. Fig. 2 shows the RL-knitted fabric which consists of only one type of loop (for the notations see e.g. [6]). The front and back view of this pattern differ from each other. Fig. 3 illustrates the RR-knitted fabric and LL-knitted fabric with their corresponding abstract representations. Both of these patterns produce the same front and back view. More general knitted fabrics are specified by an arbitrary arrangement of $\mathrm{R}$ - and L-loops. There are in fact more complex knitted fabrics which consist of more than two types of loops. For simplicity we will however concentrate our investigation on knitting patterns as described above. Due to the repetitive structure of a knitting pattern, a subdivision into basic elements can be done. There are two basic elements, one corresponding to an L-loop and one corresponding to an R-loop (Fig. 4).

Thus modeling of a knitwear pattern is reduced to modeling the structure of a knitting yarn within basic elements. In a subsequent step the knitwear pattern is then synthesized from these basic elements by taking various boundary conditions into account, that must be fulfilled. The location of the knitting yarn

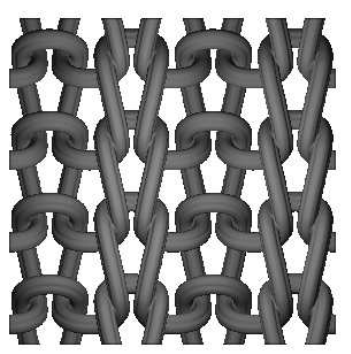

\begin{tabular}{|l|l|l|l|}
\hline & $\mathbf{R}$ & & $\mathbf{R}$ \\
\hline & $\mathbf{R}$ & & $\mathbf{R}$ \\
\hline & $\mathbf{R}$ & & $\mathbf{R}$ \\
\hline & $\mathbf{R}$ & & $\mathbf{R}$ \\
\hline
\end{tabular}
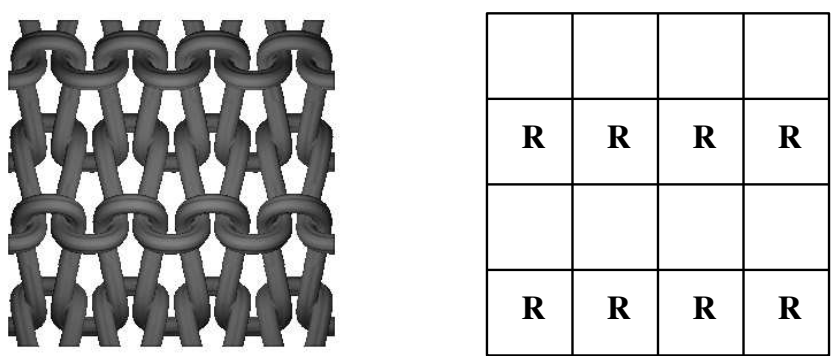

Fig. 3. RR-knitted fabric, LL-knitted fabric.

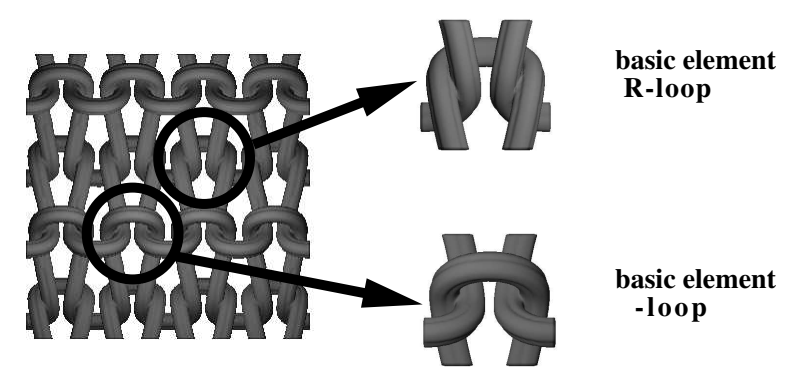

Fig. 4. Knitted fabric and basic elements.

within a basic element is defined by four pieces $C_{1}, C_{2}, C_{3}, C_{4}$ of a parametrized $3 \mathrm{D}$ skeleton curve $\mathrm{C}(\mathrm{t})$. The four pieces together can be considered to define a single knitting loop and are parametrized as shown in Fig. 5. A curve-length parameterization is done so that the following conditions hold: $C\left(t_{i}\right)=p_{i}$, $t_{0}=0, t_{1}=t_{2}=0.25, t_{3}=0.5, t_{4}=t_{5}=0.75$ and $t_{6}=1.0$. The parameterization of the skeleton curve affects properties of the yarn structure, e.g. twisting behavior, and must be chosen to ensure continuous transitions of the yarn and its microstructure between adjacent basic elements.

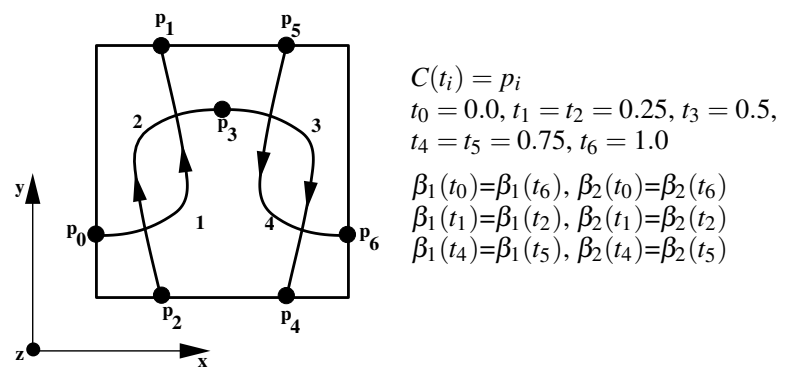

Fig. 5. Parameterization of skeleton curve $\mathrm{C}=<C_{1}, C_{2}, C_{3}, C_{4}>$ within a basic element.

The skeleton curve $\mathrm{C}$ has various symmetry characteristics: $C_{1}$ is basically a rotated version of $C_{2}$ and $C_{3}$ is a rotated version of $C_{4}$. Reflecting $C_{2}$ at a plane parallel to the $(\mathrm{y}, \mathrm{z})$-plane 
produces $C_{3}$. The same relationship holds for $C_{1}$ and $C_{4}$. Furthermore, the skeleton curve of an R-loop is the same as the reflection (at the xy-plane) of the skeleton curve of an L-loop (Fig. 5). The skeleton curve $\mathrm{C}$ determines the location of the thread course. We will deal with the definition of the yarn microstructure and its positioning in the next section.

\section{Modeling OF the YARn Microstructure}

Knitting yarn typically consists of a large number of thin fibers. A fiber may be of different materials like cotton, silk or nylon and it has a much greater length than thickness. Combining a certain number of fibers, usually by applying a twisting operation, results in a level-one yarn. A level-two yarn is produced by joining level-one yarns accordingly (Fig. 6). For a detailed description on fabrication and properties of yarns see for example [1], [6], and [11].

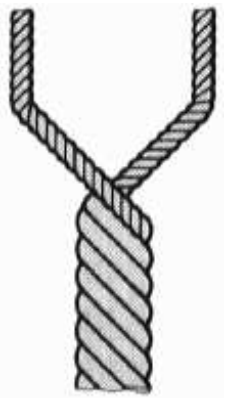

(a)

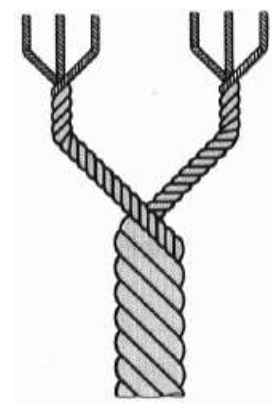

(b)
Fig. 6. Level-one yarn (a) and level-two yarn (b) [EHM+93].

A yarn has a complex microstructure due to the large number of constituting fibers and their thinness. As the diameter of a single fiber is in the order of micrometers, it has a very low opacity and is not perceivable individually. Only the collection of fibers and their spatial arrangement determines the visual impression of a yarn. A geometric model with the representation of each single fiber was considered to be too costly and unnecessarily detailed for the generation of the visual appearance of the yarn structure. Additionally severe aliasing problems have to be expected when using a geometric model for representing the microstructure of knitting yarn.

We therefore decided to model the yarn as a volume data set. A density value thereby reflects the frequency of fibers in a certain region of a basic element which makes up a knitted fabric. The volume data of a basic element is generated by specifying a $2 \mathrm{D}$ cross-section of a knitting yarn. This cross-section is swept along the skeleton curve whereby one or more additional rotations produce the twisted shape of the yarn.

Fig. 7 shows 2D cross-sections of different yarn types. Density values correspond to fiber frequency, i.e. high density values correspond to locations with a large number of fibers. A yarn cross-section usually consists of one or more circular regions of high density where most of the fibers are located. Density values drop-off at the boundary of the circular regions which is due to the fact that fibers are less numerous in these regions. Scattered spots of high density (see Fig. 7(b)(c)) correspond to fibers or bundles of fibers that are detached from the main strand of the

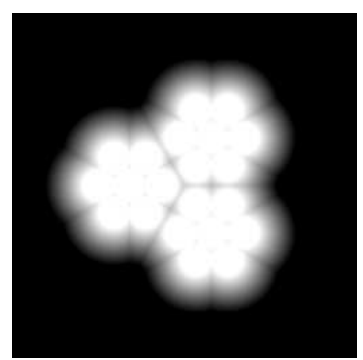

(a) Smooth three-ply yarn.

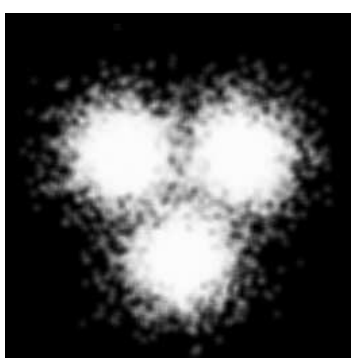

(b) Rough three-ply yarn.

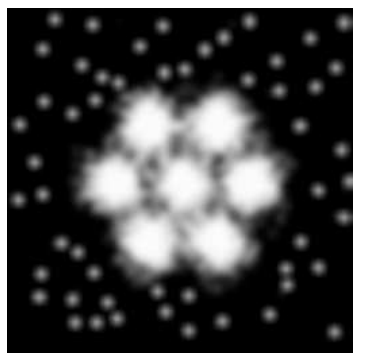

(c) Seven-ply yarn

Fig. 7. Cross-sections of different yarn types.

yarn. They are essential for the fleecy and soft appearance of a knitting yarn.

Various parameters determine the structure of yarn cross-sections: number and size of circular regions; drop-off rate when approaching the boundary of the circular regions; distribution, size and density of scattered spots. Varying these parameters allows for modeling different types of yarns. Due to the statistical distribution of single fibers within regions of high density, the modeling of these structures was performed by employing a probability distribution. We used a rotational symmetric distribution function, which was translated in correspondence with the centers of individual subyarns. By specifying the distribution along the radius of a circular region, different characteristics of a yarn can be simulated. According to the chosen distribution, a large number of points in $\Re^{2}$ are generated. These points are taken to define a footprint with associated $\alpha$-values depending on the distance to the center. By blending together these footprints, we obtain the final cross-sections. The cross-sections of Fig. 7(b) and Fig. 7(c) were produced by using a Gaussian distribution function. In Fig. 7(c) some additional high density spots are superimposed in order to simulate larger groups of fibers being farther away from the yarn center.

Sweeping a yarn cross-section along the skeleton curve and producing the volume data of a basic element is done as follows (see Fig. 8): The spatial extent of a basic element is subdivided into a uniform grid. Each grid point $g$ is projected orthogonally onto the parameterized skeleton curve $\mathrm{C}$ producing a point $\mathrm{p}(\mathrm{t})$. At each point of the skeleton curve a local coordinate system $(\bar{x}(t), \bar{y}(t), \bar{z}(t))$ is defined. The $\bar{z}(t)$ - axis coincides with the tangent direction at point $\mathrm{p}(\mathrm{t})$ and the $\bar{x}(t)-, \bar{y}(t)-$ and $\bar{z}(t)$ - axes are mutually orthogonal. Grid point $g$ lies in the $(\bar{x}(t), \bar{y}(t))$-plane which also contains the 2D yarn cross-section for the determination of the density value at point $g$. The twisting behavior of a level-one yarn is taken into account by rotating point $g$ in the $(\bar{x}(t), \bar{y}(t))$-plane by a parameter dependent angle 


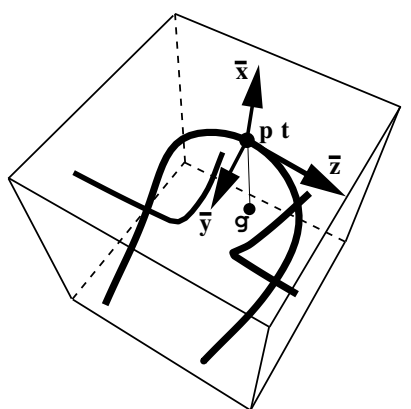

(a)

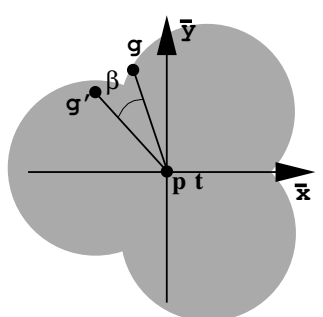

(b)

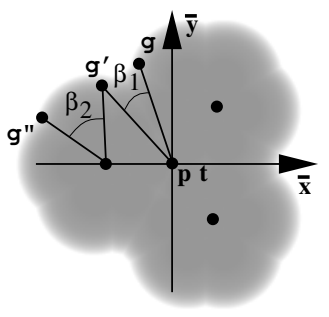

(c)

Fig. 8. (a) Volume data generation for (b) level-one and (c) level-two yarns.

$\beta_{1}(t)$. This results in a position $g^{\prime}$ where the $2 \mathrm{D}$ cross-section is sampled (see Fig. 8(b)). The transformation for level-two yarns is a little bit more complicated. A level-two yarn is constructed by putting together and twisting subyarns which are themselves made up of twisted fibers. Rotation by $\beta_{1}(t)$ around the center of the $(\bar{x}(t), \bar{y}(t))$-plane accounts for the twisting of the entire yarn. Afterwards, $g^{\prime}$ is rotated by another angle $\beta_{2}(t)$ around the center of the subyarn closest to $g^{\prime}$. This produces a position $g^{\prime \prime}$ where the yarn's cross-section is finally sampled (see Fig. 8(c)). The twisting rate between yarn and subyarns usually differs, so that $\beta_{1}(t)$ and $\beta_{2}(t)$ are typically different in magnitude. Furthermore, in most cases $\beta_{1}(t)$ and $\beta_{2}(t)$ have opposite signs, i.e. if $\beta_{1}(t)$ is rotating clockwise $\beta_{2}(t)$ is rotating counterclockwise. The twisting rate is dependent on the type of yarn and considerably influences the visual impression of a basic element. This is also true for the spatial extent of a basic element, i.e. the ratio between length, width, and height. To ensure a smooth continuation of a yarn between adjacent basic elements the local coordinate systems as well as rotation angles $\beta_{1}()$ and $\beta_{2}()$ must be the same at the positions $p_{0}$ and $p_{6}, p_{1}$ and $p_{2}, p_{4}$ and $p_{5}$ (see Fig. 5).

\section{EFFICIENT RENDERING OF KNITWEAR STRUCTURES}

Rendering of knitted fabrics is done through direct volume visualization. One approach would be to construct a volume data set of the entire knitted fabric by simply pasting together the volume data sets of the corresponding basic elements. This strategy is both, time and memory consuming. Furthermore, the highly repetitive structure of knitted fabrics as defined in previous sections can be exploited for efficient rendering. The general approach is to render only basic elements and to combine the results, so that, without loss of information, an image of the whole knitted fabric is gained. To be able to synthesize an image of the knitted fabric from images of the basic elements some restrictions must be put on the lighting and shading model, i.e. we use parallel projection and assume constant lighting direction (point light sources at infinity). We will discuss two cases: Case one deals with knitted fabrics that consist of only one type of basic element, and case two handles knitted fabrics with two types of basic elements.

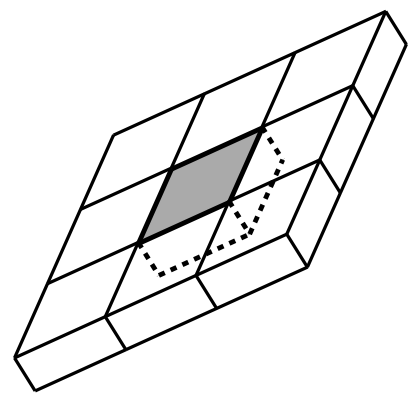

(a) Volume data set of knitted fabric.

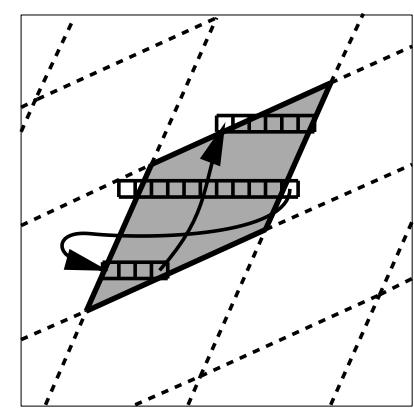

(b) Tiling of imageplane with rendered top face of the basic element.
Fig. 9. Rendering of knitted fabrics with one basic element.

When a knitted fabric consists of merely one basic element then only the top face of the basic element is rendered. Only those viewing rays piercing the top face are cast through the volume data of the basic element. This produces intensity values within a quadrilateral which is used in a subsequent step to tile the image plane (see Fig. 9). Casting rays through the volume data of a basic element must take into account that the basic element is actually part of a larger knitted fabric. Therefore, whenever a ray leaves one of the side faces of the basic element, it has to be reset cyclically to the corresponding parallel face. If for example the viewing ray leaves the basic element at position $\left(x_{\min }, y, z\right)$ it is continued at position $\left(x_{\max }, y, z\right)$ (see Fig. 10). Sampling along the ray is done at equidistant locations. This must be considered when resetting a ray. Following a viewing ray stops as soon as the ray leaves the basic element at its bottom face.

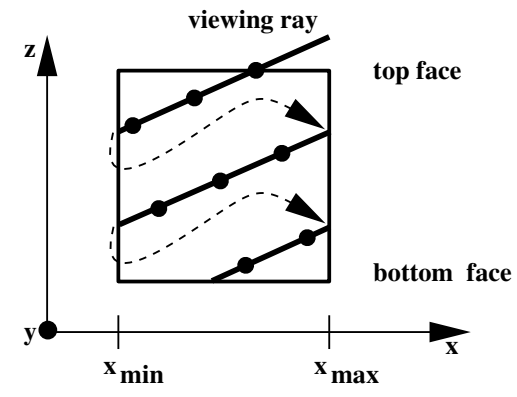

Fig. 10. Cyclic ray casting of a basic element.

The periodic structure of the knitted fabric nicely carries over to periodic patterns in the image plane. After calculating the intensities for the quadrilateral which corresponds to the top face of a basic element, a tiling of the image plane with nonoverlapping translated copies of the quadrilateral is straightforward (see Fig. 9(b)). To avoid sampling artifacts we assume, 
that all four corner points of the quadrilateral have the same relative position (e.g., pixel center) in those four pixels which contain the corner points.

When a knitted fabric is made up of two types of basic elements, rendering is done differently. Depending on the viewing direction, at most three faces of a basic element are visible. The visible faces of both basic elements are rendered, this time without the cyclic resetting of rays. In addition to the intensity, an $\alpha$-value is stored for each viewing ray. This $\alpha$-value corresponds to the length of a viewing ray within the extent of the basic element and naturally varies between different rays. After this operation there exists a hexalateral intensity image with $\alpha$-channel for each basic element (see Fig. 11). The final image of the knitted fabric is constructed by tiling the image plane with these hexalateral images. They overlap, however, and the $\alpha$-channels are used to either perform a front-to-back or a backto-front blending operation [8].

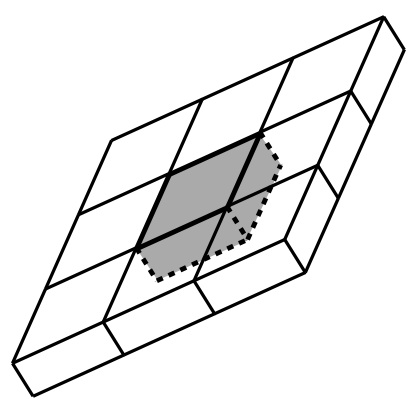

(a) Volume data set of knitted fabric.

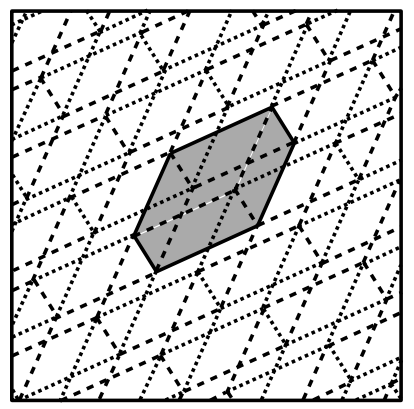

(b) Covering of imageplane with overlapping hexahedral images.
Fig. 11. Rendering of knitted fabrics with two basic elements.

This section has shown that volume visualization of periodic knitwear patterns can be reduced to rendering basic elements only. The resulting images of the basic elements can then be easily combined to generate an image of the entire knitwear pattern.

\section{RESUlTS AND IMAGES}

The research project has been realized by implementing some of the subtasks in $\mathrm{C}++$ and by using various existing software systems for certain other subtasks. Program code was written, e.g. for the generation of yarn cross-sections, for the generation of volume data sets of basic elements, and for tiling the image plane with images of basic elements. The skeleton curve within a basic element has been modelled by using the commercially available CAD-program PYTHA (cf. [10]). Finally we used VolVis [2] to volume visualize basic elements and entire knitted fabrics. The generation of the volume data for a basic element with resolution 50x30x50 takes approximately 90 seconds on an Indy-SC. The final image generation of a 100x150 resolution image takes about 40 seconds.

Plates 1-6 show different yarn types producible with our approach. In Plates 1-3 variations in the number of subyarns are presented that make up the entire yarn. Possible variations due to the representation of the yarn as volume data set are shown in Plates 4-6. These variations include modifications of color, opacity, lighting conditions, and lighting model. Plate 6 illus- trates a special type of yarn (a Mouliné yarn) which consists of differently colored subyarns.

Plates 7-9, and 16 show basic elements for the fundamental weave of knitted fabric. In Plate 7 a three-ply yarn and in Plate 8 a seven-ply yarn is used for the construction of a basic element. Only a rather low resolution $(50 \times 30 \times 40)$ of the volume data set for such an element is sufficient to produce high quality images.

Plates 10-14 present variations of RL-knitted fabric. Plates 10 and 12 show the front view of an RL-knitted fabric, and Plate 14 gives the back view of the same data set. To allow a comparison of our results with real knitwear, Plate 11 contains the scanned image of a real RL-knitted fabric. Plate 13 is the result of the simulation of a similar yarn which is given in Plate 11. It turned out that it is quite difficult to scan a knitwear pattern with a characteristical 3D-structure. Scanners, that are designed to handle 2D-objects, cannot reproduce the 3D-structure correctly. Parts of the structure, mainly those farther away from the plane of focus, appear blurred and with insufficient lighting.

Plates 15-22 illustrate the abilities of our methods and techniques to generate more complex knitted fabrics. Plates 15, 17 , 19, and 21 give an overview of RR-knitted fabrics, LL-knitted fabrics and variations thereof. Plates 16,18,20, and 22 illustrate the $3 \mathrm{D}$ shape of these patterns by using a close-up view.

\section{CONCLusion And Future Work}

We presented a novel technique for modeling the 3D-shape of knitwear. Our methods compare favorablely with previous approaches which are based on 2D texture mapping of scanned textiles. Our method allows an explicit and flexible control of the yarn and the entire knitted fabric. Efficient rendering techniques enable a fast virtual design and rapid prototyping with high-quality representation of the results.

Future work will include mapping of knitted fabrics onto freeform surfaces. Positioning of knitwear onto a geometric model of e.g. a display dummy will further facilitate virtual design of textiles. Obviously, the rendering technique described in Section 4 will not be proper for all views of a free-form surface. For a close-up view, the rendering is still efficient since only a few basic elements have to be displayed. For views from farther away our approach can be used to generate realistic 2D textures and can then be combined with standard texture mapping techniques.

We will look into more complex knitted stitch types as well. Increasing the number of possible knitted stitch types should enable us to visualize more complex knitted fabrics like cable and chain stitches.

The capabilities of modeling yarn structures within basic elements will be augmented to allow varying yarn thickness and to include special effects yarns like knotted yarns, bouclé yarns, and higher level yarns consisting of differently colored subyarns. Furthermore, we will have to address the visualization of boundary regions of knitted fabrics and the visualization of seams when joining together different pieces of knitwear.

The physical simulation of knitwear is still very much an open problem. There has been some work done on physically simulating the drape of woven materials. The more complex thread topology of knitting materials has so far apparently prevented similar work on the physical simulation of knitwear. The loca- 
tion of a skeleton curve within a basic element depends on physical properties of the yarn structure, e.g. thickness and stiffness. Therefore, a physically accurate modeling of skeleton curves will have to be addressed as well.

\section{ACKNOWLEDGMENTS}

The authors would like to thank Herta Fasser, Alwin Gröne, and Günter Knittel for their help during the research project. The work was carried out during a research and teaching visit of the first author at the University of Tübingen with financial support of the DAAD (Deutscher Akademischer Austauschdienst). The work was supported in part by a research grant of the government of Baden-Württemberg.

\section{REFERENCES}

[1] Lisa Adebahr-Doerel and Ursula Voelker, Von der Faser zum Stoff, Textile Werkstoff- und Warenkunde. Handwerk und Technik, Dr. Felix Buechner, Hamburg, Germany, 1990.

[2] Ricardo S. Avila, Lisa M. Sobierajski, and Arie E. Kaufmann, "Volvis: A diversified volume visualization system", in Visualization 94 Proceedings, pages 31-38. IEEE Computer Society, 1994.

[3] Kenichi Anjyo, Yoshiaki Usami, and Tsuneya Kurihara, "A simple method for extracting the natural beauty of hair", in Edwin E. Catmull, editor, Computer Graphics (SIGGRAPH '92 Proceedings), volume 26, pages 111-120, July 1992.

[4] David E. Breen, Donald H. House, and Michael J. Wozny, "Predicting the drape of woven cloth using interacting particles", in Andrew Glassner, editor, Computer Graphics (SIGGRAPH '94 Proceedings), volume 28, pages 365-372, July 1994.

[5] Agnes Daldegan, Nadia Magnenat Thalmann, Tsuneya Kurihara, and Daniel Thalmann, "An integrated system for modeling, animating and rendering hair', in R. J. Hubbold and R. Juan, editors, Eurographics '93, pages 211-221, Oxford, UK, 1993. Eurographics, Blackwell Publishers.

[6] Hannelore Eberle, Hermeling Herrmann, Hornberger Marianne, Menzer Dieter, and Ring Werner, Fachwissen Bekleidung, Verlag EuropaLehrmittel, Haan-Gruiten, Germany, 1993.

[7] James T. Kajiya and Timothy L. Kay, "Rendering fur with three dimensional textures", in Jeffrey Lane, editor, Computer Graphics (SIGGRAPH '89 Proceedings), volume 23, pages 271-280, July 1989.

[8] Thomas Porter and Tom Duff, "Compositing digital images", in Hank Christiansen, editor, Computer Graphics (SIGGRAPH '84 Proceedings), volume 18, pages 253-259, July 1984.

[9] Ken Perlin and Eric M. Hoffert, "Hypertexture", in Jeffrey Lane, editor, Computer Graphics (SIGGRAPH '89 Proceedings), volume 23, pages 253-262, July 1989.

[10] PYTHA-Documentation, Version 12.1, TWS Flassig GmbH, Aschaffenburg, 1995.

[11] Samuel Raz, Flat Knitting, The New Generation, Meisenbach, Bamberg, Germany, 1991.

[12] J. Weil, "The synthesis of cloth objects", in David C. Evans and Rusell J. Athay, editors, Computer Graphics (SIGGRAPH '86 Proceedings), volume 20, pages 49-54, August 1986.

[13] Yasuhiko Watanabe and Yasuhito Suenaga, "A trigonal prism-based method for hair image generation", IEEE Computer Graphics and Applications, 12(1):47-53, January 1992.

[14] Takami Yasuda, Shigeki Yokoi, Junichiro Toriwaki, and Katsuhiko Inagaki, "A shading model for cloth objects", IEEE Computer Graphics and Applications, 12(6):15-24, November 1992.

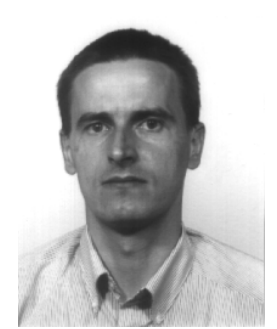

für Informatik)
Eduard Gröller is an assistant professor of the Institute of Computer Graphics at the Vienna University of Technology. His current research interests include visualization, rendering, modeling, complex dynamical systems and fractal geometry. He has graduated in Computer Science from the Vienna University of Technology in 1987 and has done Postgraduate Studies in 1988 and 1989 at the University of Kansas. In 1993 he received a PhD degree from the Vienna University of Technology. He is a member of the ACM, the IEEE Computer Society, and the GI (Gesellschaft

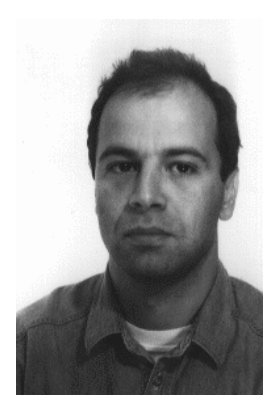

René T. Rau is a research assistant at the University of Tübingen, Germany. He received his MS in mathematics in 1991 and his PhD in 1993 from the University of Tübingen. Since 1994 he is responsible for the visualization of scientific data within a research project supported by the german science foundation (DFG). His research interests include scientific visualization, rendering, dynamic models, and geometric modeling.

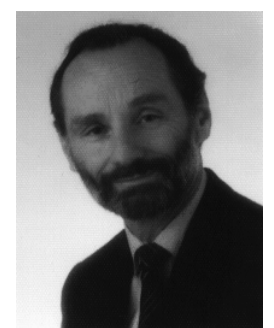

Wolfgang Straßer is a professor of Computer Science and adjunct professor of Mathematics at the University of Tübingen, Germany. His graphics group in Tübingen consists of about 20 researchers working in the areas of graphics systems design, graphics hardware, visualization, rendering and geometric modeling. He is on the editorial boards of the Computer Graphics Forum, Computers and Graphics, and CAGD. 


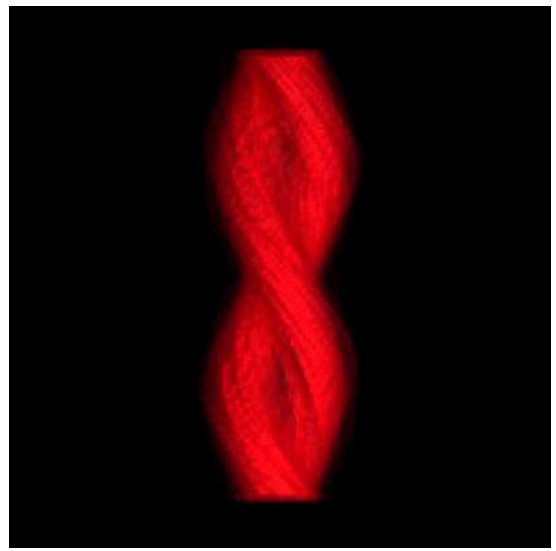

Plate 1 Two-ply yarn.

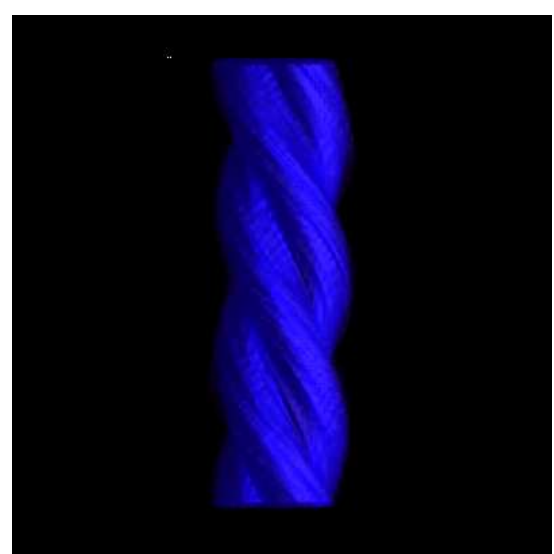

Plate 4 Three-ply yarn: modification of Plate 2 in color and opacity.

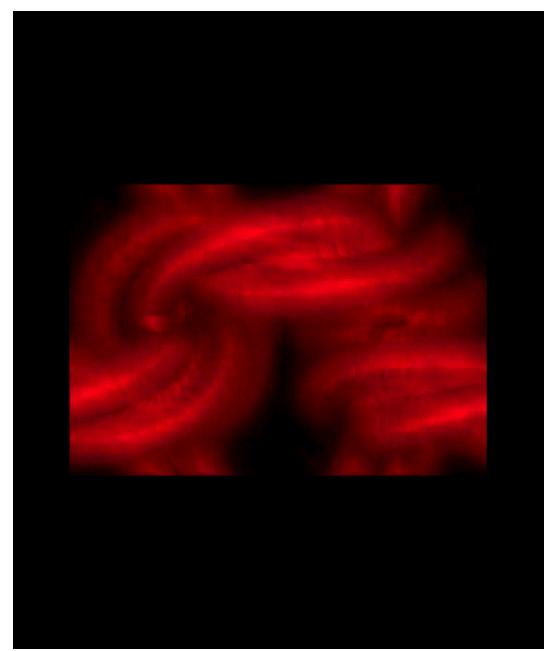

Plate 7 Basic element with a three-ply yarn (see Plate 10).

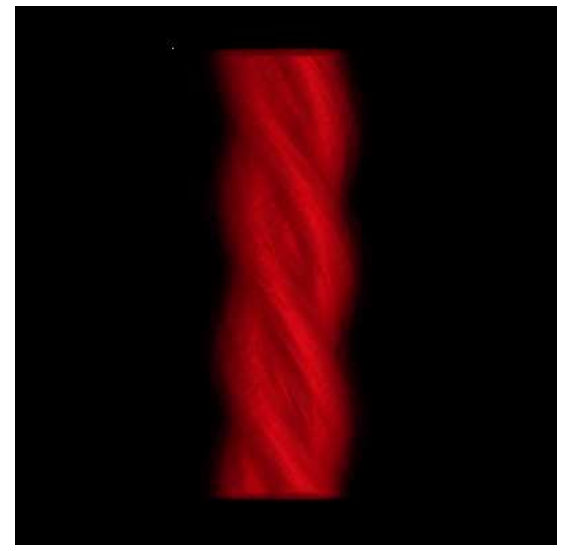

Plate 2 Three-ply yarn.

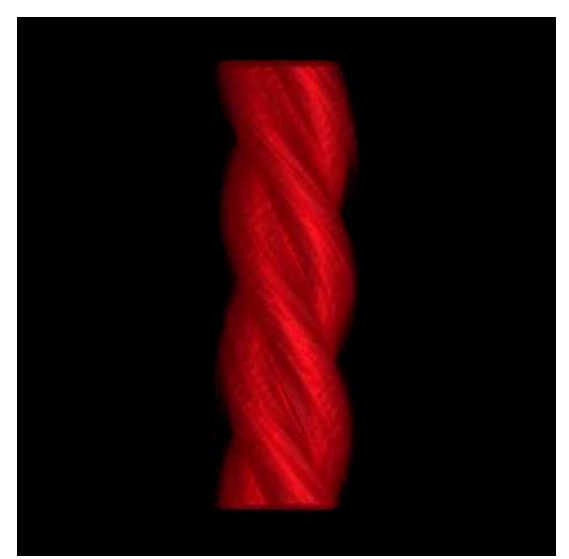

Plate 5 Three-ply yarn: modification of Plate 2 in lighting conditions, opacity and specular reflection.

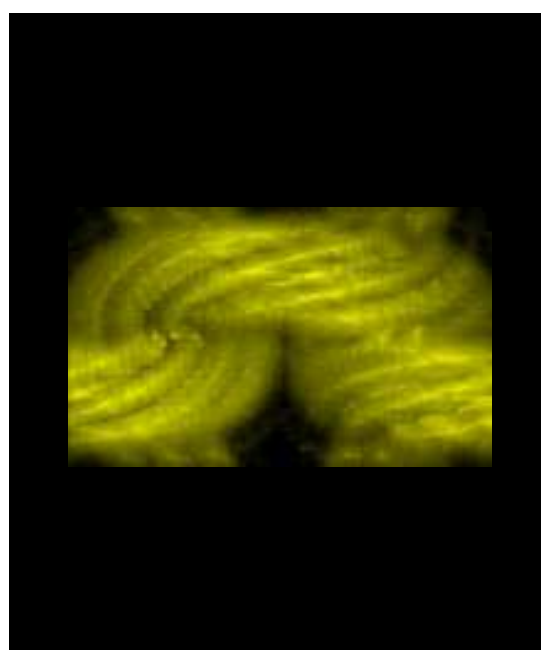

Plate 8 Basic element with a seven-ply yarn (see Plate 13).

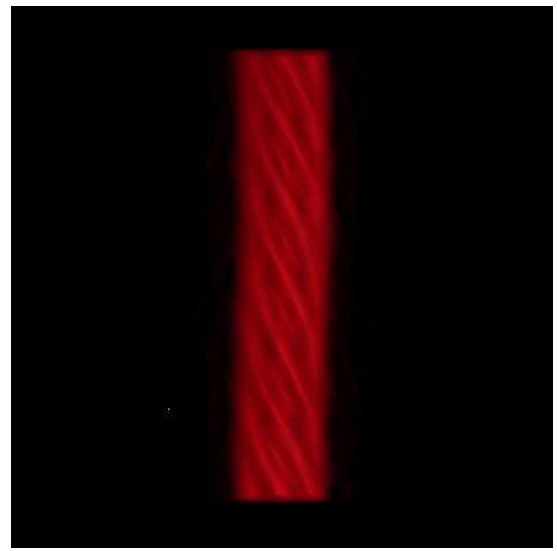

Plate 3 Seven-ply yarn.

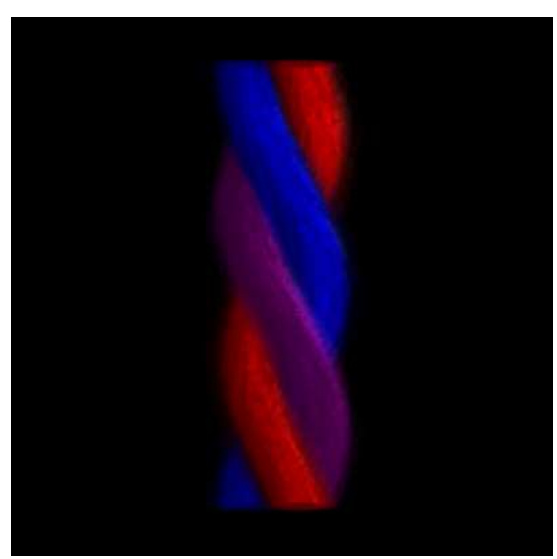

Plate 6 Mouliné yarn: yarn consists of three differently colored subyarns.

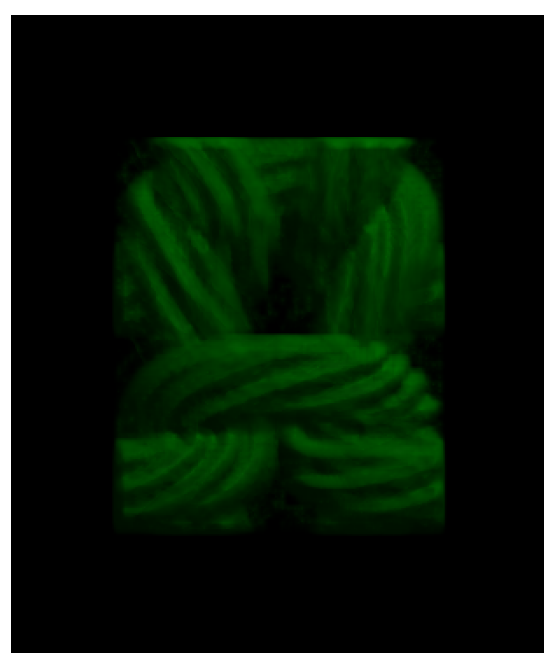

Plate 9 Basic elements for a LL knitted fabric (see Plate 15). 


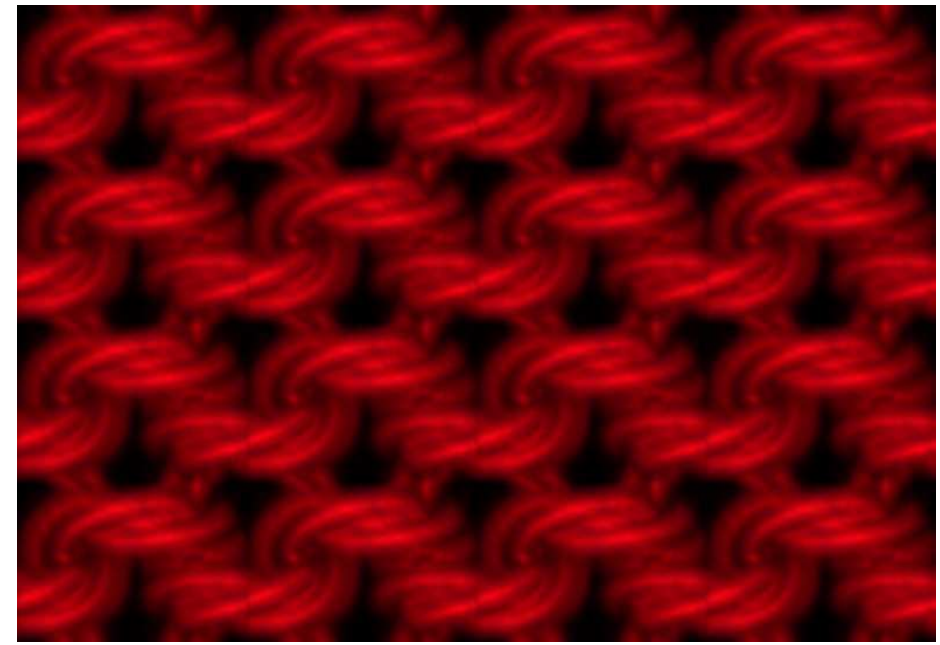

Plate 10 RL knitted fabric of a three-ply yarn generated from the basic element in Plate 7.

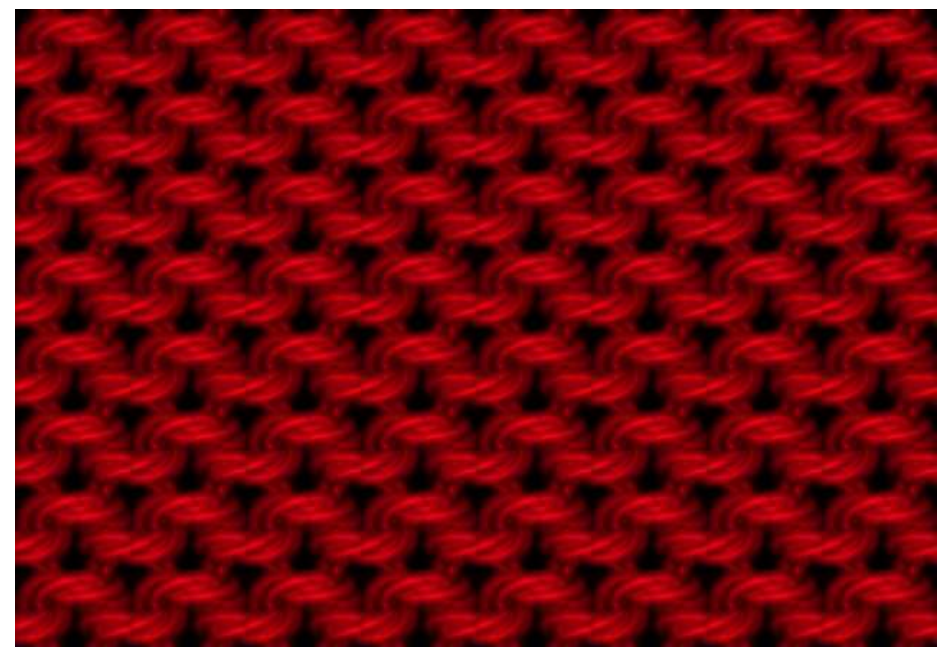

Plate 12 Less detailed view of the knitted fabric shown in Plate 10.

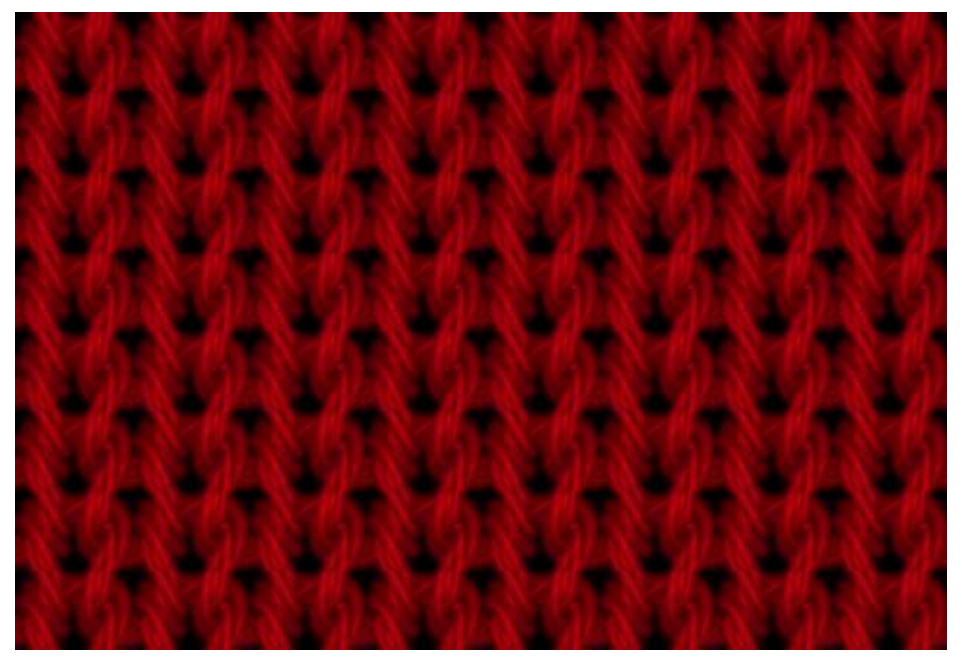

Plate 14 Back view of the knitted fabric shown in Plate 12.

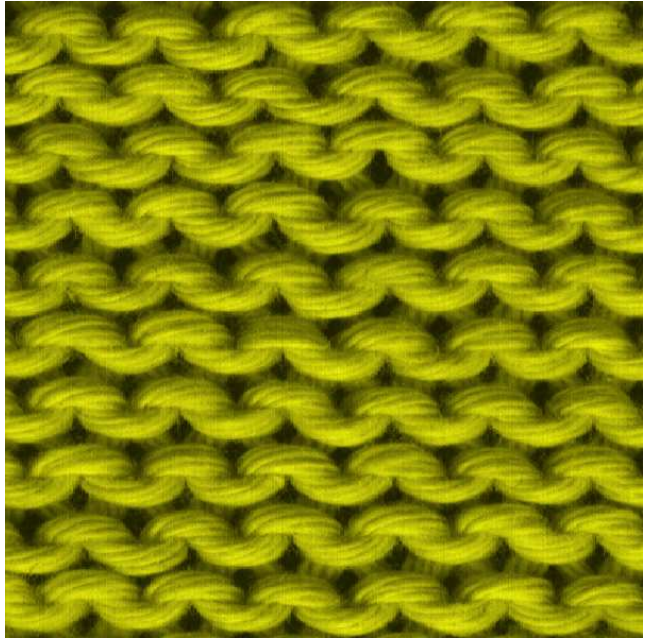

Plate 11 Scanned image of a real RL knitted fabric. See Plate 13 for the comparison with the automatically generated image.

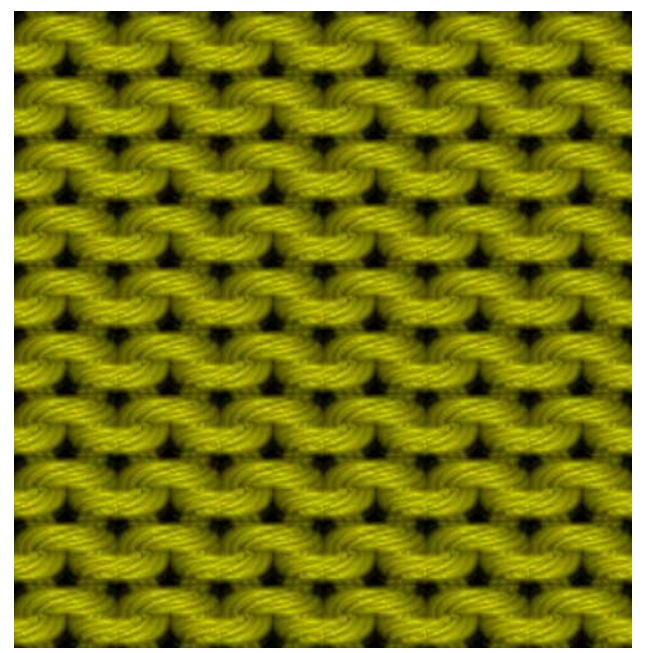

Plate 13 RL knitted fabric which simulates the scanned knitwear in Plate 11.

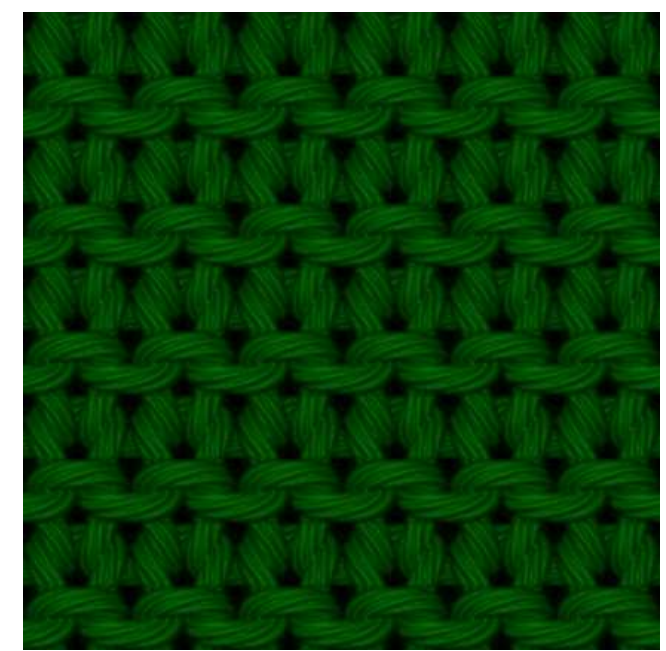

Plate 15 LL knitted fabric, basic elements see Plate 9. 


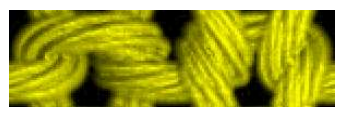

Plate 16 Basic elements of RR knitted fabric (see Plate 17)

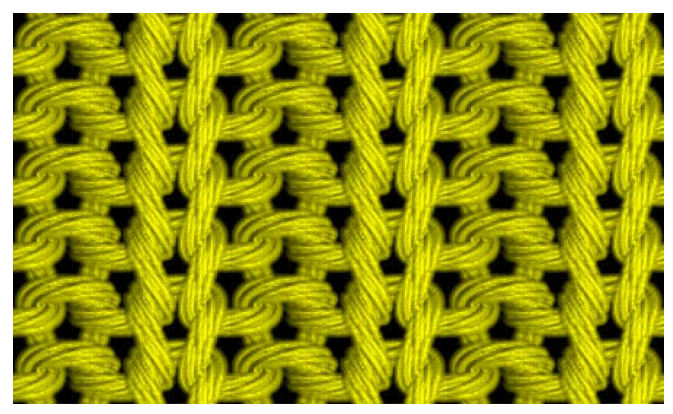

Plate 17 RR knitted fabric.

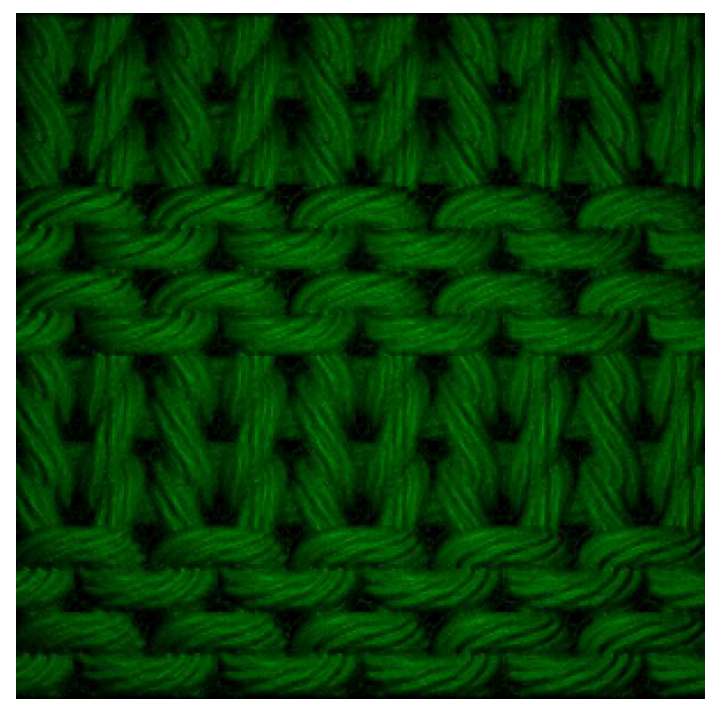

Plate 19 Variation of knitting patterns (between rows).

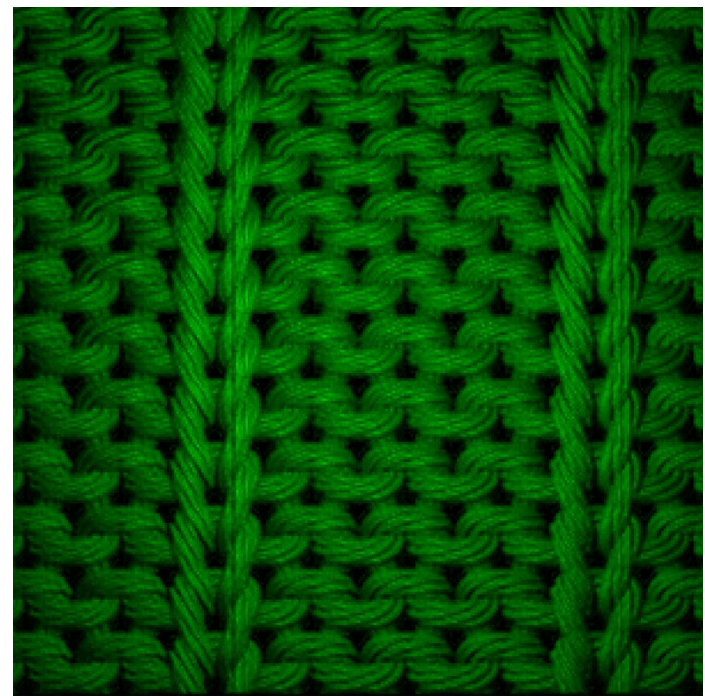

Plate 21 Variation of knitting patterns (between columns).

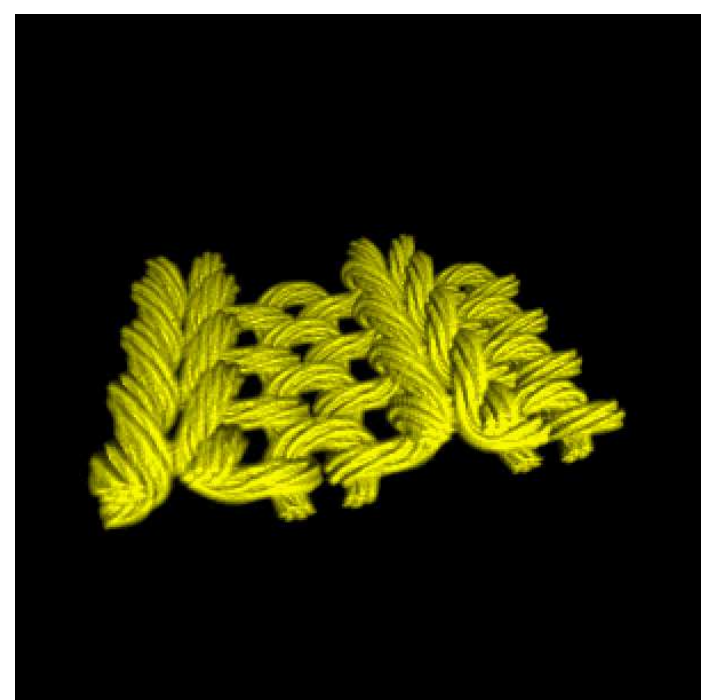

Plate 18 Perspective view of a part of the knitwear shown in Plate 17

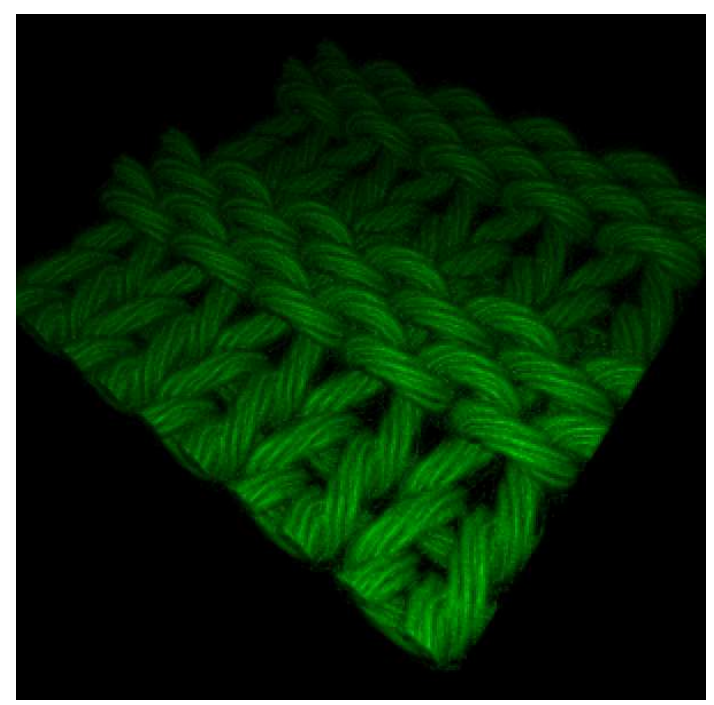

Plate 20 Perspective view of the knitwear shown in Plate 19.

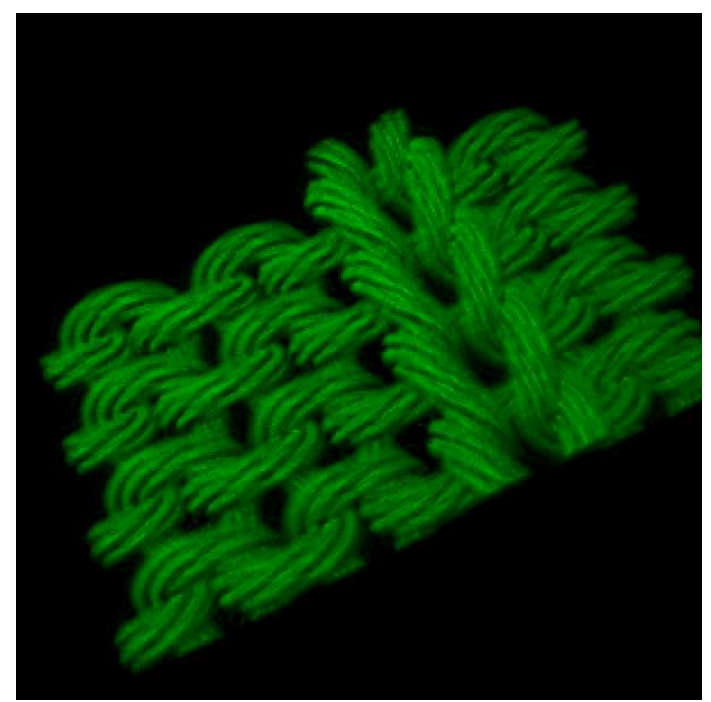

Plate 22 Perspective view of a part of the knitwear shown in Plate 21. 